Functional Neuroradiology 

Scott H. Faro - Feroze B. Mohamed Editors

Meng Law

Associate Editor

John L. Ulmer

Diffusion Tensor Imaging Section Editor

\section{Functional Neuroradiology}

Principles and Clinical Applications

说 Springer 


\section{Editors}

Scott H. Faro, MD

Professor of Radiology, Electrical and Computer

Engineering, Vice Chair of Radiology

Director of Neuroradiology, Functional Brain

Imaging Center and Clinical MRI

Temple University School of Medicine

3401 North Broad Street

Philadelphia, PA 19140-5103

USA

faros@tuhs.temple.edu

\section{Associate Editor}

Meng Law, MD

Professor of Radiology and Neurosurgery

Director of Neuroradiology

Los Angeles County Hospital

and USC Medical Center

Keck School of Medicine

University of Southern California

1520 San Pablo Street, HCC II, Suite L 1600

Los Angeles, CA 90033

USA

meng.law@usc.edu
Feroze B. Mohamed, PhD

Associate Professor of Radiology

Associate Director of Functional Brain

Imaging Center

Temple University School of Medicine

3401 North Broad Street

Philadelphia, PA 19140-5103

USA

feroze@temple.edu

Diffusion Tensor Imaging Section Editor

John L. Ulmer, MD

Professor of Radiology

Director of Neuroradiology

Medical College of Wisconsin

8701 Watertown Plank Road

Milwaukee, WI 53226

USA

julmer@mcw.edu

Additional material to this book can be downloaded from http://extras.springer.com

ISBN 978-1-4419-0343-3

e-ISBN 978-1-4419-0345-7

DOI 10.1007/978-1-4419-0345-7

Springer New York Dordrecht Heidelberg London

Library of Congress Control Number: 2011931860

(C) Springer Science+Business Media, LLC 2011

All rights reserved. This work may not be translated or copied in whole or in part without the written permission of the publisher (Springer Science+Business Media, LLC, 233 Spring Street, New York, NY 10013, USA), except for brief excerpts in connection with reviews or scholarly analysis. Use in connection with any form of information storage and retrieval, electronic adaptation, computer software, or by similar or dissimilar methodology now known or hereafter developed is forbidden.

The use in this publication of trade names, trademarks, service marks, and similar terms, even if they are not identified as such, is not to be taken as an expression of opinion as to whether or not they are subject to proprietary rights.

While the advice and information in this book are believed to be true and accurate at the date of going to press, neither the authors nor the editors nor the publisher can accept any legal responsibility for any errors or omissions that may be made. The publisher makes no warranty, express or implied, with respect to the material contained herein.

Printed on acid-free paper

Springer is part of Springer Science+Business Media (www.springer.com) 


\section{Preface}

Functional neuroradiology represents a relatively new and ever growing subspecialty in the field of neuroradiology. Neuroradiology has evolved beyond anatomy and basic tissue signal characteristics and strives to understand the underlying physiological processes of CNS disease. This new and exciting field uses a variety of advanced imaging techniques that are constantly expanding due to ongoing research in one of the most advanced fields of medicine. These techniques are initially used to characterize primarily central nervous system lesions using diffusion, perfusion, permeability, MR spectroscopy, and positron emission tomography. Following lesion characterization, localization of the eloquent cortex and key white matter structures is then obtained with state-of-the-art blood oxygen level-dependent (BOLD) fMRI and diffusion tensor imaging (DTI) techniques.

This book is a comprehensive review and offers a complete introduction to cutting edge functional imaging in neuroradiology, including the physical principles and clinical applications of these advanced techniques. This book contains 49 chapters and is divided into nine major sections.

These sections include a review of the physical principles of all key functional techniques. Lesion characterization is presented using diffusion, perfusion, permeability, MR spectroscopy, and positron emission tomography. There is an overview of BOLD fMRI physical principles and key concepts, including scanning methodologies; experimental research design; data analysis; neurovascular uncoupling and functional connectivity. After characterization of a lesion, the book follows the clinical path of localizing eloquent cortex and white matter using BOLD fMRI and DTI as part of a presurgical mapping procedure. Current and future clinical applications of BOLD fMRI in neurosurgery, neurology, psychiatry, neuropsychology, and neuropharmacology are also presented. There is an indepth review of multimodality functional imaging that follows an algorithmic approach to brain lesion characterization as well as additional chapters on common brain abnormalities such as epilepsy, traumatic brain injury, and demyelinating disease. A section on imaging techniques that are not based on proton imaging is also presented and it includes magnetoencephalography; positron emission tomography; molecular imaging; and metabolic imaging. This is followed by a section on DTI and BOLD fMRI of the spine and cerebral spinal fluid flow imaging. Finally, a full-color neuroanatomical brain atlas of eloquent cortex and key white matter tracts and a review of BOLD fMRI paradigms are presented.

The field of functional neuroradiology is young. There is a great interest and need to continue to advance this area of radiology. Functional neuroradiology represents a vital sub specialty with important clinical applications and great opportunities for clinical and cognitive 
neuroscience research. It is our hope that this book will give a thorough introduction to this new field and will be a reference, to all physicians and cognitive neuroscientists, of the current and emerging clinical applications of functional neuroradiology.

Philadelphia, PA

Scott H. Faro

Philadelphia, PA

Feroze B. Mohamed

Los Angeles, CA

Meng Law 


\section{Acknowledgements}

I want to thank my dear wife Paula for her remarkable patience and continued support in all of my projects. I would like to thank Dr. Anita Pruzan-Hotchkiss, my first scientific mentor, and long time friend who taught me that there is no such thing as a ridiculous question. I would like to thank my mentors and colleagues from my molecular biology graduate program, diagnostic radiology residency - same same, and adult and pediatric neuroradiology fellowship training programs for their encouragement of my academic pursuits. I thank Dr. Raymond Damadian, a pioneer in MRI technology, who I have been honored to get to know and who has helped to inspire my interest in MR physics. All of these individuals lead by example by their tireless enthusiasm, work ethic, academic achievements and kind nature and fostered my great interest in teaching and research. I thank, whole heartily, all of the contributors of this book, for their participation which is truly a labor of love. I am honored to call all of them my colleagues. I want to also thank Dr. Chip Jungreis and all of my neuroradiology colleagues at Temple for their support that gave me the opportunity to put this book together. I have a special thanks to Feroze Mohamed, my long time friend and colleague who is a daily inspiration and has supported me and our work for almost twenty years. I would like to thank my Sensei's Merrick and Traci and my students, domo arigato gozaima su. I also thank my parents Leonard and Alice and my brother Jeff and my sister Joan for their continued support. Eddie man, thank you, come here buddy. Lastly, I would like to thank my friends at Springer, especially Andrew Moyer, and Maureen Pierce who share our goal to produce this timely book on this exciting and important topic of the physical principles and clinical applications of functional neuroradiology.

Scott H. Faro, MD

I am extremely grateful to Shaila for being patient and supportive of this project. She was a great sounding board during the years it took to compile this book. Another person I owe my deepest gratitude is Prof. Simon Vinitski, who not only generously guided me in the research project for my doctoral work, but also inspired me to pursue academia with undeterred zeal. I also cannot but mention my parents, Mohamed and Subaidha Ali, and my brothers, without whose encouragement I would not be here writing, and compiling work for, this book. More specifically, I would like to thank the authors of the various chapters of this book. They should know that this book is essentially a reflection of their work, which, in many instances, is the result of years of research. I would be remiss if I did not mention Scott Faro, whose friendship 
and assistance have influenced a good deal of my academic work. Finally, I wish to express my appreciation to Andrew Moyer, of Springer for his coordination of the efforts of the various people that made this book possible, and to Maureen Pierce for her meticulous editorial work.

Feroze B. Mohamed, PhD

To my parents, Lawrence and Sue, for their love and inspiration.

Meng Law, MD 


\section{Contents}

\section{Part I Diffusion and Perfusion Imaging}

1 Physical Principles of Diffusion Imaging

Thinesh Sivapatham and Elias R. Melhem

2 Clinical Applications of Diffusion.

Reza Forghani and Pamela W. Schaefer

3 Physical Principles of MR Perfusion and Permeability Imaging:

Gadolinium Bolus Technique.

Mark S. Shiroishi, Saulo Lacerda, Xiaoli Tang, Naira Muradyan,

Timothy P.L. Roberts, and Meng Law

4 Non-gadolinium Perfusion Technique (Arterial Spin Labeling) Huan Tan and Jonathan H. Burdette

5 Clinical Applications of MR Perfusion Imaging. Ronald L. Wolf

6 Stroke: Clinical Application of Perfusion and Diffusion

Niloy Dasgupta and Max Wintermark

7 Clinical Applications of Dynamic Contrast-Enhanced (DCE)

Permeability Imaging

Saulo Lacerda, Mark S. Shiroishi, and Meng Law

\section{Part II Magnetic Resonance Spectroscopy}

8 Magnetic Resonance Spectroscopy: Physical Principles Stefan Blüml

9 Magnetic Resonance Spectroscopy: Clinical Applications

Alena Horská and Ivan Tkáč

Part III Multi Modality Functional Neuroradiology

10 Functional Imaging-Based Diagnostic Strategy:

Intra-axial Brain Masses

Arastoo Vossough and Seyed Ali Nabavizadeh

11 Functional Neuroimaging of Epilepsy. 
12 Functional Neuroradiology of Traumatic Brain Injury

Matilde Inglese and Eytan Raz

13 Functional MRI of Multiple Sclerosis

Heather A. Wishart

14 Functional Neuroradiology of Psychiatric Diseases

Paolo Nucifora

15 Neuroimaging of Pain

Richard H. Gracely and Pia C. Sundgren

Part IV BOLD Functional MRI: Physical Principles

16 Principles of BOLD Functional MRI

Seong-Gi Kim and Peter A. Bandettini

17 fMRI Scanning Methods

Alexander B. Pine and Feroze B. Mohamed

18 Experimental Design and Data Analysis for fMRI

Geoffrey K. Aguirre

19 Challenges in fMRI and Its Limitations

R. Todd Constable

20 Neurovascular Uncoupling in Functional MR Imaging

Jorn Fierstra and David John Mikulis

21 Functional Connectivity MR Imaging

Michelle Hampson, Xilin Shen, and R. Todd Constable

22 Clinical Challenges of Functional MRI

Nader Pouratian and Susan Y. Bookheimer

\section{Part V BOLD Functional MRI: Clinical Applications}

23 fMRI of Language Systems: Methods and Applications

Jeffrey R. Binder

24 Functional MRI Studies of Memory in Aging, Mild Cognitive Impairment, and Alzheimer's Disease

Shannon L. Risacher, Heather A. Wishart, and Andrew J. Saykin

25 fMRI Wada Test: Prospects for Presurgical Mapping of Language and Memory

Brenna C. McDonald, Jacob Kean, and Andrew J. Saykin

26 fMRI of Human Visual Pathways.

Edgar A. DeYoe, John L. Ulmer, Wade Mueller, Lotfi Hacein-Bey,

Viktor Szeder, Mary Jo Maciejewski, Karen Medler, Danielle Reitsma, and Jedediah Mathis

27 Brain Mapping for Neurosurgery and Cognitive Neuroscience Joy Hirsch

28 Pediatric Applications of fMRI

Nolan R. Altman and Byron Bernal 
29 fMRI of the Central Auditory System

Deborah A. Hall

30 fMRI of Epilepsy

Karsten Krakow

31 Applications of fMRI to Psychiatry

Melissa Lopez-Larson and Deborah A. Yurgelun-Todd

32 Applications of fMRI to Neurodegenerative Disease

Shamseldeen Y. Mahmoud, Stephen E. Jones, and Michael D. Phillips

33 Applications of MRI to Psychopharmacology

Dan J. Stein, Yihong Yang, and Betty Jo Salmeron

34 Functional MRI: Cognitive Neuroscience Applications

Mark D'Esposito, Andrew S. Kayser, and Anthony J.W. Chen

\section{Part VI Diffusion Tensor Imaging}

35 Diffusion Tensor Magnetic Resonance Imaging: Physical Principles

Thomas A. Gallagher, Andrew L. Alexander, and Aaron S. Field

36 Issues in Translating Imaging Technology and Presurgical Diffusion Tensor Imaging.

John L. Ulmer, Jeffrey I. Berman, Wade M. Mueller, Wolfgang Gaggl,

Edgar A. DeYoe, and Andrew P. Klein

37 White Matter: Functional Anatomy of Key Tracts

Mohit Maheshwari, Andrew Klein, and John L. Ulmer

38 Epilepsy: Clinical Applications of Diffusion Tensor Imaging

Timothy M. Shepherd and Christopher P. Hess

39 DTI and fMRI: Review of Complementary Techniques

Jay J. Pillai and Domenico Zaca

\section{Part VII Beyond Proton Imaging}

40 Magnetoencephalography: Epilepsy and Brain Mapping Erin Simon Schwartz and Timothy P.L. Roberts

41 PET-CT/MR Imaging in Head and Neck Cancer:

Physiologic Variations, Pitfalls, and Directed Applications

Laurie A. Loevner

42 Simultaneous PET and MR Imaging of the Human Brain

Ciprian Catana, A. Gregory Sorensen, and Bruce R. Rosen

43 The Role of Molecular Imaging in Personalized Medicine Michelle Bradbury

44 Metabolic Magnetic Resonance Imaging:

A Case for Bioscales in Medicine.

Keith R. Thulborn, Ian C. Atkinson, and Aiming Lu 
Part VIII Functional Spine and CSF Imaging

45 Functional MRI of the Spinal Cord: Diffusion-Weighted, Diffusion Tensor Imaging, and Fiber Tractography

Meng Law, Majda M. Thurnher, Eric Schwartz, and Adam Flanders

46 MRI of Hydrocephalus and CSF Velocity Imaging 949 William G. Bradley Jr.

\section{Part IX Neuroanatomical Brain Atlas}

47 Neuroanatomical Atlas of Key Presurgical and Cognitive Eloquent Cortex Regions.

Feroze B. Mohamed, Michael Yannes, Muhammed Malik, and Scott H. Faro

48 Normal Anatomic Atlas of Common White Matter Tracts Using DTI 983 Andrew P. Klein

49 fMRI Paradigms

Feroze B. Mohamed and Scott H. Faro

Index 


\section{Contributors}

Geoffrey K. Aguirre, MD, PhD Assistant Professor of Neurology, Department of Neurology, Hospital of the University of Pennsylvania, 3 West Gates Building, 3400 Spruce Street, Philadelphia, PA 19104, USA

aguirreg@mail.med.upenn.edu

Andrew L. Alexander, PhD Associate Professor, Department of Medical Physics and Psychiatry, Waisman Center, University of Wisconsin, 1500 Highland Avenue, Madison, WI 53705, USA

alalexander2@wisc.edu

Nolan R. Altman, MD Chief, Department of Radiology, Miami Children's Hospital, 3100 SW 62nd Avenue, Miami, FL 33155, USA, nolan.altman@mch.com

Ian C. Atkinson, PhD Research Assistant Professor, Center for Magnetic Resonance Research, University of Illinois Medical Center, Chicago, IL 60612, USA ian@uic.edu

Peter A. Bandettini, PhD Director, Functional MRI Core Facility, Chief, Section on Functional Imaging Methods, Laboratory of Brain and Cognition, National Institutes of Health, 10 Center Drive, Building 10, Room 1D80, Bethesda, MD 20892, USA

Bandettini@nih.gov

Jeffrey I. Berman, PhD Research Assistant Professor of Radiology, Department of Radiology, Children's Hospital of Philadelphia, University of Pennsylvania School of Medicine, 2nd Floor Wood Building, Room 2115, Philadelphia, PA 19104, USA BermanJ@email.chop.edu

Byron Bernal, MD, CCTI Clinical Neuroscientist, Department of Radiology, Miami Children's Hospital, 10529 SW 103rd Avenue, Miami, FL 33176, USA byron.bernal@mch.com

Jeffrey R. Binder, MD Professor of Neurology and Biophysics, Department of Neurology, Medical College of Wisconsin, 8701 Watertown Plank Road, MEB Room 4670, Milwaukee, WI 53226, USA

jbinder@mcw.edu

Stefan Blüml, PhD Director, New Imaging Technologies Laboratory, Associate Professor of Research Radiology, Department of Radiology, Children's Hospital Los Angeles, Keck School of Medicine, University of Southern California, MS 81, 4650 Sunset Boulevard, Los Angeles, CA 90027, USA sbluml@chla.usc.edu 
Susan Y. Bookheimer, PhD Joaquin Fuster Professor of Cognitive Neuroscience, Department of Psychiatry and Biobehavioral Sciences, David Geffen School of Medicine at UCLA, 760 Westwood Plaza, Suite 17-369, Los Angeles, CA 90095, USA Sbook@ucla.edu

Michelle Bradbury, MD, PhD Department of Radiology, Memorial Sloan Kettering Cancer Center, 1275 York Avenue, Room C-278, New York, NY 10065, USA bradburm@mskcc.org

William G. Bradley, Jr. MD, PhD, FACR Professor and Chairman, Department of Radiology, UC San Diego Health System, 200 West Arbor Drive, \#8224, San Diego, CA 92103-8224, USA

wgbradley@ucsd.edu

Jonathan H. Burdette, MD Professor, Department of Radiology, Wake Forest University School of Medicine, Medical Center Boulevard, Winston-Salem, NC 27157, USA jburdett@wfubmc.edu

Ciprian Catana, MD, PhD Department of Radiology, Athinoula A. Martinos Center for Biomedical Imaging, Massachusetts General Hospital, 149 13th Street, Suite 2.301, Charlestown, MA 02129, USA ccatana@nmr.mgh.harvard.edu

Anthony J.W. Chen, MA, MD Assistant Professor, Department of Neurology, VA Medical Center - San Francisco, Helen Wills Neuroscience Institute, University of California, San Francisco, 132 Barker Hall, Berkeley, CA 94720, USA Anthony.chen@va.gov

R. Todd Constable, PhD Professor, Director MRI Research, Co-Director Yale MRRC, Diagnostic Radiology, Biomedical Engineering, and Neurosurgery, The Anlyan Center, Yale University School of Medicine, 300 Cedar Street, New Haven, CT 06520, USA todd.constable@yale.edu

Mark D'Esposito, MD Professor of Neuroscience and Psychology, Director, Henry H. Wheeler, Jr. Brain Imaging Center, Helen Wills Neuroscience Institute, University of California, Berkeley, 132 Barker Hall, Berkeley, CA 94720-3190, USA despo@berkley.edu; http://bic.berkeley.edu/despolab/

Niloy Dasgupta, MD Department of Neuroradiology, University of Virginia, PO Box 800170, Charlottesville, VA 22902, USA

Edgar A. DeYoe, PhD Professor, Department of Cell Biology, Neurobiology and Anatomy, Medical College of Wisconsin, 8701 Watertown Plank Road, Milwaukee, WI 53226, USA

Associate Professor, Department of Biophysics, Medical College of Wisconsin, 8701 Watertown Plank Road, Milwaukee, WI 53226, USA

Department of Radiology, Medical College of Wisconsin, 8701 Watertown Plank Road, Milwaukee, WI 53226, USA

deyoe@mcw.edu

Scott H. Faro, MD Professor of Radiology, Electrical and Computer Engineering Vice Chair of Radiology, Director of Neuroradiology, Functional Brain Imaging Center and Clinical MRI, Temple University School of Medicine, 3401 North Broad Street, Philadelphia, PA 19140-5103, USA faros@tuhs.temple.edu

Aaron S. Field, MD, PhD University of Wisconsin School of Medicine and Public Health, Department of Radiology, Section of Neuroradiology,

600 Highland Avenue, Madison, WI 53792, USA afield@uwhealth.org 
Jorn Fierstra, PhD Candidate Rudolf Magnus Institute of Neuroscience, University Medical Center Utrecht, Utrecht, The Netherlands

Division of Neuroradiology, Division of Neurosurgery, Toronto Western Hospital, University Health Network, 399 Bathurst Street, Toronto, ON M5T 2S8, Canada jornfierstra@gmail.com

Adam Flanders, MD Department of Radiology, Division of Neuroradiology/ENT, Thomas Jefferson University Hospital, Suite 1080B Main Building, 132 S. Tenth St. Philadelphia, PA 19107-5244 USA adam.flanders@jefferson.edu

Reza Forghani, MD, PhD Associate Chief, Department of Radiology, Sir Mortimer B. Davis Jewish General Hospital, Montreal, QC, Canada McGill University, 3755 Cote Ste-Catherine Road, Room C-210.2, H3T 1E2, Montreal, QC, Canada rforghani@jgh.mcgill.ca

Wolfgang Gaggl, MS Graduate Student, Magnetic Resonance Biophysics, Department of Radiology, Medical College of Wisconsin, Milwaukee, WI 53226, USA wgaggl@mcw.edu

Thomas Anthony, Gallagher MD Neuroradiology Fellow, Department of Radiology, Section of Neuroradiology, University of Wisconsin School of Medicine and Public Health, 600 Highland Avenue, Madison, WI 53792, USA tgallagher@uwhealth.org

Richard H. Gracely, PhD Center for Neurosensory Disorders, School of Dentistry, University of North Carolina, 2100 Old Dental, Campus Box 7455, Chapel Hill, NC 27599-7455, USA rgracely@unc.edu

Lotfi Hacein-Bey, MD Diagnostic Radiologist, Radiological Associates of Sacramento Medical Group Inc., 1500 Expo Parkway, Sacramento, CA 95815, USA

Deborah A. Hall, BSc, PhD Professor, National Biomedical Research Unit in Hearing, Ropewalk House, 113 The Ropewalk, Nottingham, NG1 5DU, UK

Division of Psychology, School of Social Sciences, Nottingham Trent University, Nottingham, NG1 4BU, UK deb.hall@nottingham.ac.uk

Michelle Hampson, PhD Department of Diagnostic Radiology, Yale University, MRRC, TAC-N121, 300 Cedar Street, PO Box 208043, New Haven, CT 06520-8043, USA michelle.hampson@yale.edu

Christopher P. Hess, MD, PhD Assistant Professor, Neuroradiology, Neuroradiology Chief, Department of Radiology and Biomedical Imaging, San Francisco VA Medical Center, University of California, San Francisco, 505 Parnassus Avenue, Room L-358,

San Francisco, CA 94143, USA christopher.hess@ucsf.edu

Joy Hirsch, PhD Professor of Functional Neuroradiology, Neuroscience, and Psychology, Director, Functional MRI Research Center, Departments of Radiology, Neuroscience, and Psychology, Columbia University Medical Center, 710 W 168th St, B-41, Box 108, New York, NY 10032, USA joyhirsch@yahoo.com 
Alena Horská, PhD Assistant Professor, The Russell H. Morgan Department of Radiology and Radiological Science, Johns Hopkins University, 600 N. Wolfe Street, Park Building, Rm 367H, Baltimore, MD 21287, USA ahorska@jhmi.edu

Matilde Inglese, MD, PhD Associate Professor of Radiology and Neurology, Department of Radiology, New York University, 660 First Avenue, 4th floor, New York, NY 10016, USA

Matilde.Inglese@nyumc.org

Stephen E. Jones, MD, PhD Assistant Professor of Radiology, Department of Neuroradiology, Cleveland Clinic, 9500 Euclid Avenue, MC U-15, Cleveland, OH 44195, USA

Joness19@ccf.org

Andrew S. Kayser, MD, PhD Assistant Professor, Department of Neurology, Ernest Gallo Clinic \& Research Center, University of California at San Francisco, 5858 Horton Street, Suite 200, Emeryville, CA 94608, USA akayser@gallo.ucsf.edu

Jacob Kean, PhD Visiting Assistant Professor, Department of Physical Medicine and Rehabilitation, Indiana University Center for Neuroimaging, Rehabilitation Hospital of Indiana, Indiana University School of Medicine, 4141 Shore Drive, Indianapolis, IN 46254, USA

jakean@indiana.edu

Seong-Gi Kim, PhD Paul C. Lauterbur Chair in Imaging Research, Professor of Radiology and Neurobiology, Department of Radiology, University of Pittsburgh,

3025 East Carson Street, Pittsburgh, PA 15203, USA

kimsg@pitt.edu

Andrew P. Klein, MD Assistant Professor of Radiology-Neuroradiology,

Department of Radiology, Medical College of Wisconsin, 9200 West Wisconsin Avenue, Milwaukee, WI 53226, USA

aklein@mcw.edu

Karsten Krakow, MD, PhD Medical Director, Asklepios Neurologische Klinik Falkenstein, Asklepiosweg 15, Königstein, 61462, Germany

k.krakow@asklepios.com

Saulo Lacerda, MD Department of Radiology, Beneficencia Portuguesa de Sao Paulo, Rua Maestro Cardim, 769, Il Subsolo, Paraiso, Sao Paulo, SP 01323-000, Brazil,

saulolacerda@hotmail.com

Meng Law, MD Professor of Radiology and Neurosurgery, Director of Neuroradiology, Los Angeles County Hospital and USC Medical Center, Keck School of Medicine, University of Southern California, 1520 San Pablo Street, HCC II, Suite L 1600,

Los Angeles, CA 90033, USA

meng.law@usc.edu

Laurie A. Loevner, MD Professor of Radiology, Otorhinolaryngology: Head and Neck Surgery, and Neurosurgery, Division of Neuroradiology, Department of Radiology, University of Pennsylvania, 3400 Spruce Street, Philadelphia, PA 19104, USA

Laurie.loevner@uphs.upenn.edu 
Melissa Lopez-Larson, MD Assistant Professor, Psychiatry, Department of Psychiatry, The Brain Institute, University of Utah, 383 Colorow Drive, Salt Lake City, UT 84108, USA melissa.lopez-larson@hsc.utah.edu

Aiming Lu, PhD Research Assistant Professor, Center for Magnetic Resonance Research, University of Illinois Medical Center, 1801 West Taylor Street, Suite 1A, M/C 707, OCC, Chicago, IL 60612, USA aiminglu@uic.edu

Mary Jo Maciejewski, PhD Postdoctoral Research Fellow, Center for Brain Health, University of Texas at Dallas, 2200 W. Mockingbird Lane, Dallas, TX 75235, USA mjm091000@utdallas.edu

Mohit Maheshwari, MD Assistant Professor, Department of Radiology, Medical College of Wisconsin, Children's Hospital of Wisconsin, 9000 W. Wisconsin Avenue, Milwaukee, WI 53226, USA mmaheshwari@chw.org

Shamseldeen Y. Mahmoud, MD Image Analysis Lab Co-Coordinator, Department of Radiology, Cleveland Clinic, 9500 Euclid Avenue, MC U-15, Cleveland, OH 44195, USA; Assiut University, Asyut, Egypt mahmous@ccf.org

Muhammed Malik, BS Department of Radiology, Temple University School of Medicine, 3401 North Broad Street, Philadelphia, PA 19140, USA muhammed.malik@gmail.com

Jedediah Mathis Research Technologist, Department of Radiology (Neuro Lab), Medical College of Wisconsin, 8701 Watertown Plank Road, Milwaukee, WI 53226, USA

Brenna C. McDonald, PsyD, MBA Assistant Professor of Radiology and Imaging Sciences, Department of Radiology and Imaging Sciences, Indiana University School of Medicine, 950 West Walnut Street, R2 E12Y, Indianapolis, IN 46202, USA mcdonalb@iupui.edu

Karen Medler 5th Year PhD Candidate, Neurolab, Department of Biophysics, Medical College of Wisconsin, 8701 Watertown Plank Road, Milwaukee, WI 53226, USA kmedler@mcw.edu

Elias R. Melhem, MD, PhD Vice-Chair of Academic Affairs, Department of Radiology, University of Pennsylvania, 3400 Spruce Street, Philadelphia, PA 19104, USA emelhem@rad.upenn.edu

David John Mikulis, MD Division of Neuroradiology, University Health Network: Toronto Western Hospital, University of Toronto, 399 Bathurst Street, Toronto, ON M5T 2S8, Canada mikulis@uhnres.utoronto.ca

Feroze B. Mohamed, PhD Associate Professor of Radiology, Associate Director of Functional Brain Imaging Center, Department of Radiology, Temple University School of Medicine, 3401 North Broad Street, Philadelphia,

PA 19140-5103, USA

feroze@temple.edu

Wade Mueller, MD Professor, Department of Neurosurgery, Medical College of Wisconsin, 8701 Watertown Plank Road, Milwaukee, WI 53226, USA 
Naira Muradyan, PhD Lead Senior Research Scientist, Research and Development, iCAD, Inc., 98 Spit Brook Rd, \#100, Nashua, NH 03062, USA

nmuradyan@icadmed.com

Seyed Ali Nabavizadeh, MD Research Fellow, Neuroradiology Division,

Department of Radiology, Hospital of the University of Pennsylvania, 3400 Spruce Street,

Philadelphia, PA 19104, USA

ali.nabavizadeh@uphs.upenn.edu

Paolo Nucifora, MD, PhD Assistant Professor of Radiology, Attending Neuroradiologist, Department of Radiology, Veteran's Administration Medical Center, University of Pennsylvania School of Medicine, 3400 Spruce Street, One Silverstein, Philadelphia, PA 19104, USA

paolo.nucifora@uphs.upenn.edu

Michael D. Phillips, MD Vice Chairman, Research and Academic Affairs, Imaging Institute, Department of Radiology, Cleveland Clinic, 9500 Euclid Avenue, MC U-15, Cleveland, OH 44195, USA phillim@ccf.org

Jay J. Pillai, MD Director, Functional MRI, Neuroradiology Division, The Johns Hopkins University School of Medicine, The Johns Hopkins Hospital, 600 N. Wolfe Street, Phipps Building, B-100, Baltimore, MD 21287, USA jpillai1@jhmi.edu

Alexander B. Pine, PhD Cleveland Clinic Lerner College of Medicine, 9500 Euclid Avenue, Cleveland, OH 44195, USA

Nader Pouratian, MD, PhD Assistant Professor of Neurosurgery, Neuroscience, and Biomedical Engineering, Director, Neurosurgical Movement Disorders Program, Department of Neurosurgery, University of California, 10945 Le Conte Avenue, Suite 2120, Los Angeles, CA 90095, USA

npouratian@mednet.ucla.edu

Eytan Raz, MD Department of Neurological Sciences, Sapienza University, Viale dell' Università 30, Rome, 00185, Italy

eytan.raz@uniroma1.it

Danielle Reitsma Graduate Student, Magnetic Resonance Biophysics, Department of Radiology, Medical College of Wisconsin, 8701 Watertown Plank Road, Milwaukee, WI 53226, USA

dreitsma@mcw.edu

Shannon L. Risacher, BS Department of Radiology and Imaging Sciences, Indiana University School of Medicine, IU Center for Neuroimaging, 950 West Walnut Street, R2, E124, Indianapolis, IN 46202, USA srisache@iupui.edu

Timothy P.L. Roberts, PhD Professor, Oberkircher Family Chair in Pediatric Radiology, Department of Radiology, Children's Hospital of Philadelphia, 34th Street and Civic Center Boulevard, Wood Building, Suite 2115, Philadelphia, PA 19104, USA robertstim@email.chop.edu

Bruce R. Rosen, MD, PhD Department of Radiology, Athinoula A. Martinos Center for Biomedical Imaging, Massachusetts General Hospital, 149 13th Street, Suite 2.301, Charlestown, MA 02129, USA

bruce@nmr.mgh.harvard.edu 
Noriko Salamon, MD, PhD Associate Professor of Radiology, Department of Radiology, UCLA Medical Center, 10833 Le Conte Avenue, BL-428 CHS, MC 172115,

Los Angeles, CA 90095, USA

nsalamon@mednet.ucla.edu

Betty Jo Salmeron, MD Staff Clinician, Neuroimaging Research Branch, National Institute on Drug Abuse, NIH, 251 Bayview Boulevard, Suite 200, Room 07A717, Baltimore, MD 21224, USA

bsalmeron@mail.nih.gov

Andrew J. Saykin, PsyD, ABPP/CN Center for Neuroimaging, Department of Radiology and Imaging Sciences, Indiana University School of Medicine, 950 West Walnut Street, R2 E124, Indianapolis, IN 46202, USA asaykin@iupui.edu

Pamela W. Schaefer, MD Associate Director of Neuroradiology, Associate Professor of Radiology, Department of Radiology, Massachusetts General Hospital, Harvard Medical School, Gray 273A, 55 Fruit Street, Boston, MA 02114, USA pschaefer@partners.org

Eric Schwartz, MD Department of Radiology, Shields Health Care, 65 Westgate Dr, Brockton, MA 02302, USA eschwartz@shcpo.shields.com

Erin Simon Schwartz, MD Associate Professor of Radiology, Clinical Director, Magnetoencephalography, Department of Radiology, CHOP Radiology, Children's Hospital of Philadelphia, University of Pennsylvania School of Medicine, 324 South 34th Street, Philadelphia, PA 19104-4399, USA

SchwartzES@email.chop.edu

Xilin Shen, PhD Associate Research Scientist, Department of Diagnostic Radiology, Yale University, 300 Cedar Street, New Haven, CT 06520, USA xilin.shen@yale.edu

Timothy M. Shepherd, MD, PhD Neuradiology Fellow, Department of Radiology and Biomedical Imaging, University of California, San Francisco, 505 Parnassus Avenue, Room L-358, San Francisco, CA 94143, USA

tim.shepherd@radiology.ucsf.edu

Mark S. Shiroishi, MD Assistant Professor, Division of Neuroradiology, Department of Radiology, Keck School of Medicine, University of Southern California, 1500 San Pablo Street, Second Floor Imaging, Los Angeles, CA 90033, USA mshiroishi@gmail.com

Thinesh Sivapatham, MD Fellow, Diagnostic Neuroradiology, Department of Radiology, Hospital of the University of Pennsylvania, 3400 Spruce Street, 2 Dulles, Philadelphia, PA, 19104, USA thineshsiva@yahoo.com

A. Gregory Sorensen, MD Department of Radiology, Athinoula A. Martinos Center for Biomedical Imaging, Massachusetts General Hospital, 149 13th Street, Suite 2.301, Charlestown, MA 02129, USA sorensen@nmr.mgh.harvard.edu

Dan J. Stein, MD, PhD Professor and Chair, Department of Psychiatry and Mental Health, University of Cape Town, Groote Schuur Hospital (J-2), Anzio Road, Observatory, 7925, Cape Town, South Africa dan.stein@uct.ac.za 
Pia C. Sundgren, MD, PhD Professor, Center for Medical Imaging and Physiology, Skåne University Hospital, Lund University, Getingevägen 5, Lund, 221 85, Sweden pia.sundgren@med.lu.se

Viktor Szeder, MD, PhD Clinical Fellow, Division of Stroke, Department of Neurology, Columbia University Medical Center, 630 West 168th Street, New York, NY 10032, USA

Huan Tan, MS, PhD Candidate VT-WFU School of Biomedical Engineering and Sciences, Medical Center Boulevard, Wake Forest University Health Sciences,

Winston-Salem, NC 27157, USA

htan@wfubmc.edu

Xiaoli Tang, MD Professor, Department of Radiology, Shekou People Hospital, No. 7 Industry Road, Shekou, Nanshan, Shenzhen, Guangdong, 518067, China shellytang2005@hotmail.com

Keith R. Thulborn, MD, PhD Professor of Radiology, Physiology and Biophysics, Center for Magnetic Resonance Research, University of Illinois Medical Center, 1801 West Taylor Street, Room 1307, M/C 707, Chicago, IL 60612, USA kthulbor@uic.edu

Majda M. Thurnher, MD Associate Professor of Radiology, Department of Radiology, Section of Neuroradiology and Musculoskeletal Radiology, Medical University of Vienna, University Hospital Vienna, Waehringer Guertel 18-20, Vienna, 1090, Austria majda.thurnher@meduniwien.ac.at

Ivan Tkáč, PhD Center for Magnetic Resonance Research, University of Minnesota, 2021 Sixth Street SE, Minneapolis, MN 55455, USA ivan@cmrr.umn.edu

John L. Ulmer, MD Professor of Radiology, Director of Neuroradiology, Medical College of Wisconsin, 8701 Watertown Plank Road, Milwaukee, WI 53226, USA julmer@mcw.edu

Arastoo Vossough, PhD, MD Assistant Professor of Radiology, Department of Radiology, Children's Hospital of Philadelphia, Hospital of the University of Pennsylvania, 324 South 34th Street, CHOP Wood Building, Suite 2115, Philadelphia, PA 19104, USA arastoo.vossough@uphs.upenn.edu

Max Wintermark, MD Associate Professor of Radiology, Neuroradiology, Neurosurgery and Biomedical Engineering, Chief of Neuroradiology, Department of Neuroradiology, University of Virginia, PO Box 800170, Fedex: 1215 Lee Street-New Hospital 1st Floor, Room 1011, Charlottesville, VA 22902, USA Max.Wintermark@virginia.edu

Heather A. Wishart, PhD Brain Imaging Laboratory, Department of Psychiatry, Dartmouth Medical School, One Medical Center Drive, Lebanon,

NH 03756, USA

Heather.A.Wishart@dartmouth.edu

Ronald L. Wolf, MD, PhD Associate Professor of Radiology, Department of Radiology, Neuroradiology Section, University of Pennsylvania Health System, 3400 Spruce Street, Dulles 219, Philadelphia, PA 19041, USA ronald.wolf@uphs.upenn.edu

Yihong Yang, PhD Chief, FMRI Section, Neuroimaging Research Branch, National Institute on Drug Abuse, NIH, 251 Bayview Boulevard, Suite 200, Room 07A709, Baltimore, MD 21224, USA yihongyang@mail.nih.gov 
Michael Yannes, BS Department of Radiology, Temple University School of Medicine, 1316 W. Ontario Street, Philadelphia, PA 19140, USA

michael.yannes@gmail.com

Deborah A. Yurgelun-Todd, PhD Professor, Department of Psychiatry, The Brain Institute, University of Utah, 383 Colorow Drive, Salt Lake City, UT 84108, USA deborah.yurgelun-todd@hsc.utah.edu

Domenico Zaca, PhD Postdoctoral Fellow, Department of Radiology, The Johns Hopkins University School of Medicine, 600 N. Wolfe Street, B-115, Baltimore, MD 21205, USA dzaca2@jhmi.edu 
